\title{
Potential of the Pentacam in Screening for Narrow Angles in Patients with Chronic Angle-Closure Glaucoma
}

\author{
Seth Lartey, ${ }^{1,2}$ Abdul-Kabir Mohammed, ${ }^{3}$ Emmanuel Appiagyei, ${ }^{2}$ Kojo Akuffo $^{3}$ \\ ${ }^{1}$ EENT Department, Kwame Nkrumah University of Science and Technology \\ ${ }^{2}$ Eye Department, Komfo Anokye Teaching Hospital \\ ${ }^{3}$ Department of Optometry, Kwame Nkrumah University of Science and Technology
}

Correspondence to: Dr. Seth Lartey; email: sylartey@yahoo.com

Received: 02 October 2019; Revised: 01 May 2020; Accepted: 30 May 2020; Available online: 21 June 2020

\begin{abstract}
Background: Chronic angle-closure glaucoma (CACG) is a visually destructive disease. Effective management of CACG requires identifying eyes with narrow angle. Objective: To compare pentacam with gonioscopy in detecting narrow angles in eyes with CACG. Method: We enrolled 101 eyes with glaucoma. Gonioscopy was performed on all eyes. Using Shaffer's grading, subjects were classified into angle closure and open angle. Anterior chamber volume (ACV) and anterior chamber depth (ACD) were measured with the pentacam. Receiver operating curve was constructed for each parameter and the area under the curve (AUC) was calculated. Results: Ten eyes (9.9\%) were classified as angle closure on gonioscopy. To detect narrow angles, ACV (AUC 0.956; 95\% confidence interval (CI) 0.8940.987) performed similar to ACD (AUC $=0.930$, $\mathrm{p}=0.33$ ). Using a cutoff of $102 \mathrm{~mm}^{3}$, ACV had $100 \%$
\end{abstract}

sensitivity and $88.5 \%$ specificity for detecting narrow angles in CACG patients. With an ACV cutoff of 102 $\mathrm{mm}^{3}$, the PPV for detecting angle closure was $48.9 \%$ (95\% CI, 34.8-68.2), while the NPV was 100\% (94.1$100 \%$ ), using $9.9 \%$ prevalence of angle closure from this study. Conclusion: ACV and ACD measured by the pentacam have the potential to determine narrow angles in eyes with CACG.

Keywords: Chronic angle-closure glaucoma, Pentacam $\mathrm{HR}$, Anterior chamber volume, Gonioscopy

Ann Afr Surg. 2021; 18(1):39-44

DOI: http://dx.doi.org/10.4314/aas.v18i1.8

Conflicts of Interest: None

Funding: None

(C) 2021 Author. This work is licensed under the Creative Commons Attribution 4.0 International License.

\section{Introduction}

Glaucoma is a group of diseases that affect the optic nerve of the eye. It is characterized by a specific optic neuropathy associated with visual field defect (1). Glaucoma causes irreversible blindness worldwide and accounts for $15 \%$ of blindness in Africa (2). Compared with the rates of blindness in primary open-angle glaucoma (POAG), studies show that the risk of bilateral blindness is three times higher in primary angle-closure glaucoma (PACG) (3). Based on clinical observations, PACG is classified into two: the acute, symptomatic phase which is dramatic but occurs in only a minority of those with PACG, and the chronic, asymptomatic form which predominates (4). Chronic angle-closure glaucoma (CACG) occurs as a result of gradual and silent closure of the anterior chamber angle, which eventually results in increased intraocular pressure and, finally, damage to the optic nerve (5).

Currently, gonioscopy remains the reference standard for assessing anterior chamber angle (ACA). However, this technique requires a proficient examiner to provide 
a confident diagnosis and involves contact with the surface of the eye (6). This necessitates anesthetic agents and can cause artifacts. And even among experienced examiners, there is variability in angle grading due to the subjective nature of the assessment (7). In recent years, a Scheimpflug camera, Pentacam, has been developed to evaluate anterior chamber characteristics. The Oculus Pentacam operates on the principle of Scheimpflug imaging. It eradicates the challenges that are encountered with goniolens (8). The Oculus Pentacam provides relevant and reproducible bio data from the anterior corneal surface to the posterior lens surface in a single scan without contacting the cornea (9). However, it is unable to visualize the most peripheral part of the iris and automatically calculates the assumed apex of the ACA (10). The concordance between gonioscopy and pentacam has been found to be very good (10).

This study aimed to compare the diagnostic ability of the pentacam and gonioscopy to detect narrow angles in patients with chronic angle-closure glaucoma.

\section{Methods}

This was a hospital-based cross-sectional study. Consecutive glaucoma patients aged 40 years and above receiving treatment at the Komfo Anokye Teaching Hospital during the period of December 2017 to April 2018 were recruited for the study. All subjects received clinical examination including visual acuity, slit-lamp biomicroscopy, pentacam examination, Goldmann applanation tonometry, and gonioscopy, in that order at the glaucoma clinic. Data received from the glaucoma clinic were recorded on a data collection sheet for each subject. Glaucoma patients who have had any form of ocular surgery, congenital and secondary glaucoma, and corneal disorders, and patients who had any form of ocular infection at the time of study were excluded from the study.

\section{Pentacam examination}

The pentacam measurement was taken by a trained optometrist masked to the gonioscopy results of participants. The Pentacam HR (Typ 70900 COculus 2013, Oculus Optikgerate GmbH, Germany), device takes pictures of the anterior chamber by a rotating
Scheimpflug camera. The camera illumination system consists of a blue light-emitting diode which is ultraviolet free with a 475-nm wavelength. The rotating camera process takes pictures in three dimensions (Figure 1) and can also measure the center of the cornea precisely. The machine can take 50 meridional sections through the center of the cornea. This allows the system to align with the central thinnest point of the cornea at each section before it constructs the corneal image, and thus any eye movement is eliminated during the exam. The whole measurement process does not take more than 3 seconds (11). The participants were asked to focus on the blue fixation target without any compensation for their refractive error. When the target was focused, the rotating Scheimpflug camera captured 50 images automatically around the optical axis of the eye. The following parameters were recorded from the pentacam overview: anterior chamber angle (ACA, degrees); anterior chamber depth from the endothelium $(\mathrm{ACD}, \mathrm{mm})$; anterior chamber volume $\left(\mathrm{ACV}, \mathrm{mm}^{3}\right)$ and central corneal thickness $(\mathrm{CCT}, \mu \mathrm{m})$. The ACV is calculated using an integral calculus, which considers the anterior chamber as a solid bounded by the posterior surface of the cornea (12-mm diameter around the corneal apex), the iris, and the lens (12). The Pentacam ACA measurements were not used in this study, as their reliability in eyes with narrow angles has been questioned due to the inability of the pentacam to visualize the most peripheral part of the iris and base of the ACA (6).

\section{Gonioscopy}

Gonioscopy was performed by an ophthalmologist masked to the pentacam results of the subject. It was performed using a Goldman 3-mirror lens using standard examination technique in a moderately dark room. A topical anesthetic and hydroxyethyl cellulose were applied before examination. ACA was graded using Shaffer's grading system (13). Subjects with grade $\leq 1$ in 2 or more quadrants were classified as angleclosure whereas subjects with grade $2-4$ in 2 or more quadrants without indentation were classified as having open-angle. If necessary, the presence and extent of 
peripheral anterior synechia (PAS) were confirmed by compression gonioscopy.

If both eyes were eligible for the study, one eye was chosen at random for analysis. Study data were entered into Microsoft Excel 2013. Statistical analysis was done using IBM ${ }^{\circledR}$ SPSS ${ }^{\circledR}$ Statistics for Windows, version 23.0 and MedCalc version 19.2.1 (MedCalc Software). Clinical characteristics of the study population were expressed in mean values and standard deviation. As data were not normally distributed, Mann-Whitney Utest for unpaired data was used to determine differences between groups. Sensitivity, specificity, positive predictive value (PPV), negative predictive value (NPV) and the receiver operating characteristic (ROC) curves for pentacam parameters were calculated using gonioscopy as the reference. PPV and NPV were calculated using a prevalence of $9.9 \%$ from this study. Optimum cutoff levels were determined using the Youden index (J) $\mathrm{J}=($ sensitivity + specificity -1$)$ (14). The parameter value with the maximum Youden index was used as the cutoff value.

Ethical approval was sought from the Committee on Human Research, Publication \& Ethics (CHRPE) of the Kwame Nkrumah University of Science and Technology, School of Medical Sciences with reference number CHRPE/AP/172/18. A written informed consent was taken from all participants before enrollment.

\section{Results}

A total of 101 eyes (50 right and 51 left) were selected. This included eyes of 47 males (46.5\%) and 54 females $(53.5 \%)$. Mean age of the study population was $57.79 \pm 13.50$ years (range $40-87$ years). Mean intra ocular pressure (IOP) was $22.18 \pm 8.25 \mathrm{mmHg}$ and mean visual acuity in $\operatorname{LogMAR}$ was $1.14 \pm 1.21$. The mean corneal thickness at the thinnest locale was $508 \pm 41 \mu \mathrm{m}$. Ten eyes $(9.9 \%)$ were classified as having chronic angle closure on gonioscopy (Shaffer $\leq 1$ in 2 or more quadrants with or without PAS).

Mean ACV was $84.70 \pm 15.34$ (range $58-102$ ) $\mathrm{mm}^{3}$ in the CACG group and $140.78 \pm 37.94$ (range $79-321$ ) $\mathrm{mm}^{3}$ in the POAG group. Corresponding values for ACD were $2.61 \pm 0.33$ (range 2.04-2.93) $\mathrm{mm}$ and $3.43 \pm 0.54$ (range
1.85-5.50) mm. ACA values were $30.58 \pm 7.35$ (range

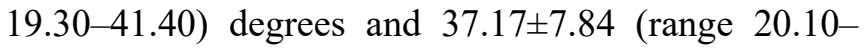
58.70) degrees. Differences between pentacam parameters in the two groups were statistically significant $(\mathrm{p}<0.05)$. Table 1 describes the clinical characteristics of the study population.

ROC curves for predicting narrow angles were constructed for ACV and ACD from the pentacam, using gonioscopy as the reference (Figure 2). Comparing the sensitivity and sensitivity at different values of ACV, we found that using a cutoff of $102 \mathrm{~mm}^{3}$, ACV had $100 \%$ sensitivity and $88.5 \%$ specificity in detecting narrow angles with area under the curve $(\mathrm{AUC})=0.956(95 \%$ confidence interval $(\mathrm{CI}), 0.894$ 0.987). Although ACV had a higher AUC than ACD (AUC $=0.930 ; 95 \% \mathrm{CI}, 0.860-0.972)$, the difference was not significant $(\mathrm{p}=0.33)$. Table 2 presents the cutoff values and the corresponding sensitivity, specificity, PPV and NPV for ACV and ACD.

With an ACV cutoff of $102 \mathrm{~mm}^{3}$, the PPV for detecting angle closure was $48.9 \%$ (95\% CI, 34.8-68.2), while the NPV was 100\% (95\% CI, 94.1-100\%), using 9.9\% prevalence of angle closure from this study.

\section{Discussion}

Chronic angle-closure glaucoma usually characterized by a silent closure of the ACA leads to severe optic nerve damage and visual field loss. Laser peripheral iridotomy is prophylactically recommended as treatment and prevention for narrow and occludable angles. However, in doing this it is imperative to identify people with narrow or occludable angles (15).

Gonioscopy is clinically the gold standard for identifying occludable angles. It is valuable and timetested clinical test to diagnose the type of glaucoma. However, its subjective nature of grading and lack of defined cut-off points between open and closed angles make comparison and follow-up difficult (16).

Dynamic gonioscopy using four-mirror gonioprism can deepen the narrow angle due to applied force, leading to false assessment of the angle especially when there is PAS. Due to the contact nature of the procedure, the possibility of spread of ocular infections and corneal abrasions has been considered during gonioscopy. 
PENTACAM IN CHRONIC ANGLE CLOSURE GLAUCOMA

Table 1: Clinical characteristics of the study population

\begin{tabular}{|c|c|c|c|c|}
\hline Parameter & Total Subjects $(n=101)$ & CACG $(n=10)$ & POAG $(n=91)$ & $\mathrm{p}$ value \\
\hline Age & $57.79 \pm 13.50(40-87)$ & $67.20 \pm 10.20(53-80)$ & $56.76 \pm 13.45(40-87)$ & $0.019^{\mathrm{a}}$ \\
\hline Gender & & & & $0.076^{\mathrm{b}}$ \\
\hline Male & 47 & 2 & 45 & \\
\hline Female & 54 & 8 & 46 & \\
\hline Visual acuity (LogMAR) & $1.14 \pm 1.21(0.00-4.00)$ & $1.50 \pm 0.47(0.00-4.00)$ & $1.01 \pm 1.00(0.00-4.00)$ & $0.137^{\mathrm{c}}$ \\
\hline IOP (mmHg) & $22.19 \pm 8.25(8.00-60.00)$ & $24.97 \pm 6.70(13.55-43.65)$ & $21.90 \pm 8.20(8.00-60.00)$ & $0.172^{\mathrm{c}}$ \\
\hline $\operatorname{ACV}\left(\mathrm{mm}^{3}\right)$ & $135.0 \pm 39.7(58.0-321.0)$ & $84.7 \pm 15.3(58-102)$ & $140.8 \pm 38.0(79-321)$ & $<0.001^{\mathrm{c}}$ \\
\hline $\mathrm{ACD}(\mathrm{mm})$ & $3.33 \pm 0.59(1.85-5.50)$ & $2.61 \pm 0.33(2.04-2.93)$ & $3.43 \pm 0.54(1.85-5.50)$ & $<0.001^{\mathrm{c}}$ \\
\hline ACA (degrees) & $36.48 \pm 8.02(19.30-58.70)$ & $30.58 \pm 7.35(19.30-41.40)$ & $37.17 \pm 7.84(20.10-58.70)$ & $<0.020^{\mathrm{c}}$ \\
\hline CTmin (um) & $508.2 \pm 41.2(408.0-615.0)$ & $474.9 \pm 41.29(40-535)$ & $511.1 \pm 39.31(434-615)$ & $<0.05^{\mathrm{c}}$ \\
\hline
\end{tabular}

Abbreviations: CACG, chronic angle-closure glaucoma; POAG, primary open-angle glaucoma; IOP, intra ocular pressure; ACV, anterior chamber volume; ACD, anterior chamber depth; ACA, anterior chamber angle; CTmin, corneal thickness at the thinnest locale. ${ }^{\text {a }}$ Independent samples t-test, ${ }^{\mathrm{b}}$ Chi-square test, ${ }^{\mathrm{c}}$ Mann-Whitney U-test, Data represent the mean $\pm \mathrm{SD}$ (range), except for gender.

Table 2: Sensitivity, specificity, positive predictive value (PPV), negative predictive value (NPV), for Pentacam parameters compared with gonioscopy for identifying narrow angles and area under the receiver operating characteristic curve (AUC) for parameters measured with the pentacam in detecting narrow angles using gonioscopy as the reference standard.

\begin{tabular}{|c|c|c|c|c|c|c|}
\hline Parameter & Cutoff & Sensitivity & Specificity & PPV & NPV & AUC \\
\hline & & $(95 \% \mathrm{CI})$ & $(95 \% \mathrm{CI})$ & $(95 \% \mathrm{CI})$ & $(95 \% \mathrm{CI})$ & $(95 \% \mathrm{CI})$ \\
\hline \multirow[t]{2}{*}{$\mathrm{ACV}$} & $\leq 102 \mathrm{~mm}^{3}$ & 100 & 88.51 & 48.9 & 100 & 0.956 \\
\hline & & $(69.2-100)$ & (79.9-94.3) & $(34.8-63.1)$ & $(94.1-100)$ & $(0.894-0.987)$ \\
\hline \multirow[t]{2}{*}{$\mathrm{ACD}$} & $\leq 2.93 \mathrm{~mm}$ & 100 & 85.06 & 42.4 & 100 & 0.930 \\
\hline & & $(69.2-100.0)$ & $(75.8-91.8)$ & $(30.8-54.8)$ & $(94.1-100)$ & $(0.860-0.972$ \\
\hline
\end{tabular}

\section{OCULUS - PENTACAM Overview}
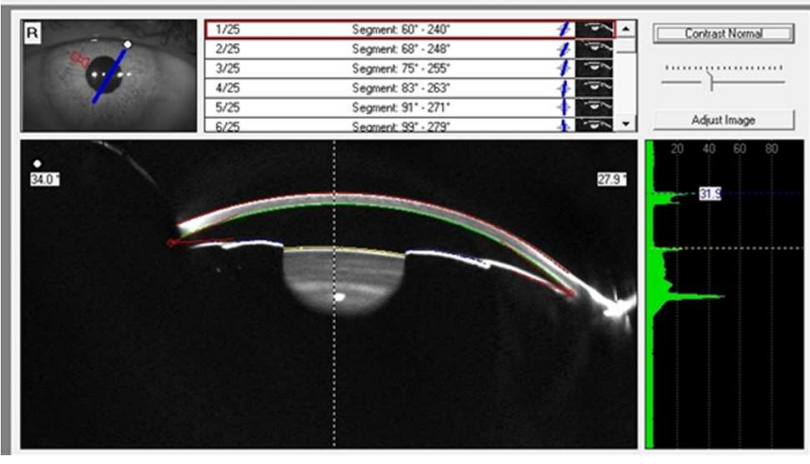

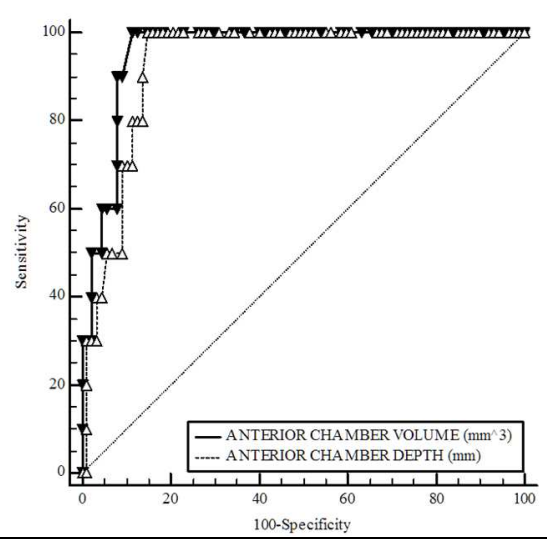

Figure 1: Three-dimensional image representation of the anterior segment obtained by the Pentacam (Oculus, Wetzlar, Germany). Note that the ACV is reduced with a corresponding quantitative value (Red box). Figure 2: Receiver Operating Characteristic curve for ACV and ACD by Pentacam

In the quest to overcome these weaknesses, several noncontact methods of angle assessment have been developed. Ultrasound biomicroscopy (UBM), anterior segment optical coherence tomography (ASOCT) and Scheimpflug imaging devices (Pentacam) are objective methods of assessing the anterior segment parameters $(17,18)$. The efficacy of UBM for evaluating the ACA is well established; however, the procedure is cumbersome and operator dependent and thus not suitable for quick assessment on a large scale (10). ASOCT is a promising 
non-contact imaging tool of angle assessment, but its efficacy in screening is not yet established (19).

Pentacam is a non-contact tool that objectively evaluates the anterior chamber with a non-significant interobserver variability (20). It is less expensive than UBM and ASOCT. It provides quantitative, reproducible and repeatable assessment of anterior segment structures (20,21).

In the current study, we compared the discriminating ability of the pentacam parameters (ACV and ACD) in detecting angle closure using gonioscopy as the reference. The ACV had a high discriminating ability $(\mathrm{AUC}=0.956)$ in screening for occludable angles with a sensitivity of $100 \%$ and specificity of $88.5 \%$ at a cutoff of $102 \mathrm{~mm}^{3}$. Corresponding ACD values were AUC $=0.930$, sensitivity of $100 \%$, and specificity of $85.1 \%$ at a cutoff of $2.95 \mathrm{~mm}$.

Several studies have compared the discriminating ability of pentacam parameters and other objective anterior segment assessment devices using gonioscopy as the reference.

Kurita et al. in 2009 examined 32 Japanese patients to assess the potential of the pentacam for screening eyes with a narrow angle (in comparison with UBM and gonioscopy) (6). The authors suggested that ACD and ACV could be used to screen patients and stated that "eyes with primary angle closure (PAC) and primary angle-closure suspect (PACS) were effectively screened out with an ACD of $2.58 \mathrm{~mm}$." Eyes with PAC and PACS were most effectively screened out with an ACD threshold of $2.58 \mathrm{~mm}$ with a sensitivity of $100 \%$ and a specificity of $87.1 \%$ (6).

Grewal et al. compared pentacam with AS-OCT (22). Grewal et al. did not consider ACA measurements in their analysis and found that ACV had the highest discriminating ability (cutoff value: $113 \mathrm{~mm}^{3}$; AUC $=0.93$, sensitivity $=90 \%$ and specificity $=88 \%$ ), outperforming AS-OCT parameters in detecting narrow angles (22).

Pakravan et al. in their study revealed that eyes with $\mathrm{ACV} \leq 100 \mu \mathrm{L}$ can be considered at high risk of acute angle closure (AAC) with sensitivity of $93.3 \%$ and specificity of $100 \%$. ACD $\leq 2.1 \mathrm{~mm}$ was another considerable risk factor for development of AAC with sensitivity of $86.7 \%$ and specificity of $100 \%$. Corresponding values for ACA $\leq 26^{\circ}$ were $73.3 \%$ and $88.2 \%$ respectively. Also, any eye that meets all of these three criteria $\left(\mathrm{ACV} \leq 100 \mu \mathrm{L}, \mathrm{ACA} \leq 26^{\circ}\right.$ and $\mathrm{ACD} \leq 2.1$ $\mathrm{mm}$ ) could also be considered at high risk with sensitivity of $66.7 \%$ and specificity of $100 \%$ (23).

The above studies showed high sensitivity and specificity values for pentacam parameters in detecting narrow angles, as revealed in the current study. Our data pointed out high AUC ROC curves for pentacam parameters and confirm previous observations about $\mathrm{ACD}$ and ACV.

We hypothesize that the differences in cutoff values reported in these studies and the current study is because of the different definition of narrow angles and the study population used in these studies. Kurita et al. defined narrow angle as eyes having an ACA width of Shaffer grade $0-$ II and limited to PAC and PAC suspect patients. Whereas in our study limited to CACG patients, ACA width of Shaffer grading $0-\mathrm{I}$ was defined as narrow angle.

Comparing different studies might be difficult since different definitions are used to classify narrow angles and also ethnicity differs: our study refers to Africans, those of Grewal et al. to Indians, and those of Kurita et al. to Japanese subjects.

Our study has several limitations; the study group was not population based and had a relatively small sample size of 101 eyes. Validating these findings in a more diverse population-based study would be useful. Although the reported sensitivity values in our study is $100 \%$, none of the specificity values recorded was in the high 90s, which should be the ideal characteristic of a diagnostic device for screening purposes (24). We did not verify the reproducibility of pentacam measurements, as this has been established $(20,21)$.

Although repeatability of ACV measurements from the pentacam has been demonstrated with an intraclass correlation coefficient of 0.991 , there are concerns that as ACV is reported with no decimal places, it may be affected by rounding error (25). 


\section{Conclusion}

ACV and ACD measured by the pentacam have the potential to determine eyes with chronic angle-closure glaucoma. It has the advantage of objectivity, repeatability and quantification. These criteria can be helpful when deciding to proceed for laser peripheral iridotomy in chronic angle-closure cases.

\section{References}

1. Foster PJ, Buhrmann R, Quigley HA, et al. The definition and classification of glaucoma in prevalence surveys. $\mathrm{Br} \mathrm{J}$ Ophthalmol. 2002; 86:238-42.

2. Resnikoff S, Pascolini D, Etya'ale D, et al. Global data on visual impairment in the year 2002. Bull World Health Organ. 2004; 82:844-51.

3. Quigley H, Broman AT. The number of people with glaucoma worldwide in 2010 and 2020. Br J Ophthalmol. 2006; 90:2627.

4. Dandona L, Dandona R, Mandal P, et al. Angle-closure glaucoma in an urban population in southern India. The Andhra Pradesh eye disease study. Ophthalmol. 2000; 107:1710-16.

5. Wright $\mathrm{C}$, Tawfik MA, Waisbourd M, et al. Primary angleclosure glaucoma: An update. Acta Ophthalmol. 2016; 94:21725.

6. Kurita N, Mayama C, Tomidokoro A, et al. Potential of the Pentacam in screening for primary angle closure and primary angle closure suspect. J Glaucoma. 2009; 18(7):506-12.

7. Smith SD, Singh K, Lin SC, et al. Evaluation of the anterior chamber angle in glaucoma: A report by the American Academy of Ophthalmology. Ophthalmology. 2013; 120(10):1985-97.

8. Chen D, Lam AKC. Reliability and repeatability of the Pentacam on corneal curvatures. Clin Exp Optom. 2009; 92:110-8.

9. Domenéch B, Mas D, Ronda E, et al. Repeatability and concordance of the Pentacam system. Comparative study of corneal parameters measured with Pentacam and Atlas. Opt Pura y Apl. 2009; 42:51-60.

10. Rossi GCM, Scudeller L, Delfino A, et al. Pentacam sensitivity and specificity in detecting occludable angles. Eur J Ophthalmol. 2012; 22:701-8.

11. Sung Y, Chung S, Nam SM. Novel technique to measure horizontal corneal diameter using a Scheimpflug image on the Pentacam. Contact Lens Anterior Eye. 2016; 39(3):234-8.

12. Oculus. Pentacam Instruction Manual: Measurement and evaluation system for the anterior eye segment. Oculus: Wetzlar, Germany, 2008.

Available at: https://docplayer.net/59508485-Pentacaminstruction-manual-measurement-and-evaluation-system-forthe-anterior-chamber-of-the-eye.html
13. Asbury T. Stereoscopic manual of gonioscopy. Am J Ophthalmol. 2014; 55(3):662.

14. Perkins NJ, Schisterman EF. The inconsistency of 'Optimal' cutpoints obtained using two criteria based on the receiver operating characteristic curve. Am J Epidemiol. 2006; 163:6705.

15. Kansara S, Blieden L, Chuang A, et al. (2016) 'Effect of laser peripheral iridotomy on anterior chamber angle anatomy in primary angle closure spectrum eyes'. J Glaucoma. 25(5): 46974.

16. Esmaeili A, Barazandeh B, Ahmadi S, et al. Assessment of the anterior chamber parameters after laser iridotomy in primary angle close suspect using pentacam and gonioscopy. Int $\mathrm{J}$ Ophthalmol. 2013; 6:680-4.

17. Radhakrishnan S, Goldsmith J, Huang D, et al. Comparison of optical coherence tomography and ultrasound biomicroscopy for detection of narrow anterior chamber angles. Arch Ophthalmol. 2005; 123:1053-9.

18. Shankar H, Taranath D, Santhirathelagan CT, et al. Anterior segment biometry with the Pentacam: Comprehensive assessment of repeatability of automated measurements. J Cataract Refract Surg. 2008; 34:103-13.

19. Mansouri K, Sommerhalder K, Shaarawy T. Prospective comparison of ultrasound biomicroscopy and anterior segment optical coherence tomography for evaluation of anterior chamber dimensions in European eyes with primary Angle closure. Eye. 2010; 24:233-9.

20. Buehl W, Stojanac D, Sacu S, et al. Comparison of three methods of measuring corneal thickness and anterior chamber depth. Am J Ophthalmol. 2006; 141:7-12.

21. Rabsilber TM, Khoramnia R, Auffarth GU. Anterior chamber measurements using Pentacam rotating Scheimpflug camera. J Cataract Refract Surg. 2006; 32:456-9.

22. Grewal DS, Brar GS, Jain R, et al. Comparison of Scheimpflug imaging and spectral domain anterior segment optical coherence tomography for detection of narrow anterior chamber angles. Eye 2011; 25(5):603-11.

23. Pakravan M, Sharifipour F, Yazdani S, et al. Scheimpflug imaging criteria for identifying eyes at high risk of acute angle closure. J Ophthalmmic Vis Res. 2012; 7:111-7.

24. Stamper RL. Glaucoma screening. J Glaucoma. 1998; 7:149-50

25. Georgios L, Maria G, Andreas K, et al. Anterior chamber volume measurements with Visante optical coherence tomography and Pentacam: repeatability and level of agreement. Clin Experiment Ophthalmol. 2009; 37:772-4. 